\title{
FAMIGLIA O FAMIGLIE NEL TERZO MILLENNIO
}

\author{
ANNAMARIA CAMPANINI \\ Università di Parma (Italia).
}

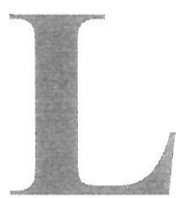

\section{FAMIGLIA O FAMIGLIE?}

a famiglia negli ultimi decenni è stata oggetto di particolare attenzione, da angolature diverse: la si è considerata come istituzione in declino (David Cooper, 1971 parlava di morte della famiglia), se ne sono analizzati i cambiamenti, se ne è riscoperta la dimensione di vitalità e di risorsa.

Certamente il gran parlare che se ne è fatto e che se ne continua a fare, gli studi a livello sociologico, ma anche psicologico ed economico, testimoniano come questo nucleo di forme sociali primarie sia di vitale importanza.

Le ragioni di tale interesse possono essere ritrovate nel fatto che la famiglia ha la proprietà di non essere mai uguale a se stessa.

Gli antropologi hanno messo in evidenza il rapporto famigliacultura, sottolineando la necessità di abbandonare criteri universalistici per abbracciare un relativismo culturale negli studi della famiglia.

Ma questo criterio, che introduce comunque una linearità di rapporto (ogni diversità è giustificata dal contesto sociale condiviso in cui la famiglia è inserita) non è più da considerarsi valido in quanto le ricerche più recenti documentano come all'interno di uno stesso contesto sociale si trovino diverse forme di famiglia.

E' possibile, infatti rilevare come in ogni assetto tipico di società si ritrovi una pluralità diversificata di forme familiari che rispondono a logiche differenziate, sia rispetto alla sub-cultura simbolica di riferimento, sia rispetto alla classe sociale. Non solo, ma la famiglia si modifica nel corso della storia modellandosi a seconda delle caratteristiche della società che la circonda, in una logica di tipo circolare: si allarga e si restringe acquisendo o perdendo funzioni, assumendo caratteristiche di maggiore o minore stabilità.

Alcune analisi estremamente interessanti, compiute a livello sociologico in Italia, sottolineano come la differenza in ampiezza e 
varietà delle strutture familiari in Italia permase fino a tutta la prima metà del XX secolo, come conseguenza anche del lento e tardivo processo di industrializzazione e come i primi segnali di discontinuità nei comportamenti familiari possano farsi risalire proprio a questi anni (Saraceno, 1998).

La rivoluzione sessuale, un non più esclusivo orientamento verso i figli, una nuova importanza attribuita al benessere ed alla felicità della coppia, la crescita dell'instabilità coniugale e infine il cambiamento del ruolo e della posizione sociale delle donne nella società sono fenomeni che, intrecciandosi alle profonde trasformazioni a livello di stratificazione sociale, di sviluppo economico e di modelli di consumo, si rafforzano a vicenda, producendo cambiamenti profondi nel modo di fare famiglia.

L'assunto di base su cui si è costituita buona parte della sociologia della famiglia, che nel passaggio da società semplici e da formazioni storico sociali tradizionali a società complesse e formazioni storicosociali moderne, la famiglia da estesa diventa nucleare modificandosi sia nella struttura che nelle funzioni, se considerato come prodotto assoluto di una istanza evoluzionistica, evidenzia tutta la sua debolezza.

Ogni formazione storico-sociale è caratterizzata dalla compresenza di più forme familiari, alcune delle quali possono essere quantitativamente più incidenti, e in questo senso è indubbio che attualmente la famiglia modale è la famiglia nucleare, ma è anche vero che molteplici risultano essere le forme di organizzazioni del quotidiano che si esternano nella famiglia (Donati - Di Nicola, 1989). Inoltre, se risulta evidente la diffusione della famiglia nucleare in tutte le classi sociali, si può tuttavia riscontrare la presenza di diverse strutture relazionali che hanno a che fare con i livelli di scolarizzazione, i rapporti col mercato del lavoro, le immagine simboliche di famiglia che sono state interiorizzate e la pluralizzazione degli stili di vita.

Si può quindi affermare che oggi sia opportuno non parlare più di famiglia, ma di famiglie, "assumendo una prospettiva pluralista che ha nella molteplicità delle specificità familiari il suo punto di riferimento"(Fruggeri, 1998).

Al di là dell'utilizzo di questa forma plurale, è comunque necessario riflettere su un problema che partendo dalla dimensione linguistica, va a toccare il sistema dei significati.

A quale entità si attribuiscono le caratteristiche di famiglia e in particolare a quale forma di famiglia viene attribuita l'etichetta di "normale"? Se si sposa una prospettiva normativa le "nuove famiglie" 
sono considerate e trattate come forme deficitarie o devianti rispetto a quella tradizionale, mentre in una prospettiva pluralista esse sono considerate e trattate semplicemente come forme differenti (Fruggeri 2001), evitando di vedere, di leggere, e quindi di confondere la diversità con la patologia.

Nel mondo occidentale l'idea di famiglia è inscindibilmente legata a quello di nuclearità e questo fa sì che manchino termini per designare i componenti di configurazioni familiari che derivino, ad esempio, da una seconda unione o da un affidamento familiare.

La complessa articolazione tra legami biologici e legami simbolici e tra relazioni e interazioni familiari, dà infatti vita a nuove connessioni e intrecci non sostituendo o cancellando quelli precedenti. (ad esempio una madre che ha figli da due partner diversi si trova a sviluppare riferimenti biologici e simbolici diversi).

Risulta quindi fondamentale l'adozione di una logica che si orienti verso la multinuclearità, modificando quindi i tradizionali confini individuati tra famiglia e ambiente, nel senso di assumerne la dimensione di interfaccia più che quella di barriera.

Tutto questo ha implicazioni notevoli da un punto di vista operativo in quanto richiede un impegno non più solo in relazione a processi di svincolo, enucleazione e individuazione, ma anche di intreccio, connessione e collegamento.

Afferma Fruggeri "...la complessità e l'articolazione delle nuove famiglie non possono essere contenute nel ristretto repertorio delle storie componibili sul canovaccio della famiglia nucleare. Le storie a forma triadica e trigenerazionale, costituite intorno alla famiglia nucleare, sono infatti insufficienti a descrivere processi in cui la linea trigenerazionale si ramifica in più di due direzioni e in cui i personaggi di una triade sono sempre contemporaneamente implicati in un'altra" (Fruggeri 2001, pg.19).

\section{ALCUNE CHIAVI DI LETTURA DELLE FAMIGLIE}

Come le famiglie, anche le teorie sulla famiglia si modificano in relazione ai cambiamenti sociali, talvolta in modo drastico in periodi di tempo molto brevi. Nei primi anni lo studio della famiglia ha portato i ricercatori a concentrare la loro attenzione su schemi e mappe per descrivere "il mondo" che si stava scoprendo. La famiglia è stata vista: come sistema di relazioni che si costituiscono sia all'interno, tra $\mathrm{i}$ membri, sia all'esterno con l'ambiente sociale; sotto l'aspetto evolutivo, 
considerandone i cicli vitali, sotto quello organizzativo, analizzandone la struttura o ancora attraverso la metafora del gioco familiare. Successivamente, venne data grande rilevanza al fatto che queste descrizioni vengono svolte da un osservatore non neutrale né oggettivo (Von Foerster, 1981, Maturana H., Varela F., 1980). L'osservatore partecipa e influenza l'oggetto fin dal momento in cui sceglie, decide arbitrariamente che quello e non altro è ciò che merita di essere preso in considerazione. In un secondo momento "lo contamina" selezionando ciò che è pertinente e ciò che è irrilevante, infine, descrivendolo con un linguaggio che è frutto del suo modo di classificare il mondo. Faremo pertanto riferimento a concetti che devono essere considerati come metafore utili per capire e non realtà oggettivamente vere.

Parleremo pertanto di organizzazioni familiari intendendole come strutture che i soggetti adottano per rapportarsi con sé stessi, con gli altri e con il mondo, costruendo modalità relazionali che danno origine a storie più o meno condivise.

La famiglia può essere considerata come un gruppo con storia, un sistema aperto autoregolantesi e, come tale, sottoposto alle proprietà dei sistemi precedentemente descritte.

Va però sottolineato come 1'attenzione dell'osservatore non debba rimanere concentrata solamente nel descrivere ed analizzare la famiglia come unità. Una sottolineatura esasperata delle regolarità, ripetitività, prevedibilità dei comportamenti familiari, rischia di sviluppare una logica di tipo unidirezionale in cui l'individuo risulta essere determinato dal gruppo familiare, proprio nel momento in cui si propone teoricamente un rapporto circolare. Fruggeri (1997, p.71) sottolinea come gli studi sul rapporto tra conoscenza e comportamento sociale, mettano in rilievo il fatto che "il comportamento interpersonale dei membri della famiglia non è dunque una semplice risposta a ciò che gli altri fanno, ma una funzione dei significati che vengono autonomamente attribuiti a tali azioni". A partire da questa considerazione si individuano tre livelli di analisi strettamente interconnessi tra loro: il livello personale, il livello familiare e il livello sociale. Ogni componente della famiglia dà senso alla propria esperienza e agisce nelle relazioni con gli altri a partire da un insieme di premesse e credenze personali che derivano dalla sua specifica posizione nel gruppo, dalle esperienze vissute precedentemente o nei rapporti con l'esterno, ma anche la famiglia, sviluppa un sistema di premesse o credenze condivise che costituiscono un contesto simbolico che dà senso all'esperienza individuale e coniuga e organizza i comportamenti dei membri della famiglia sia all'interno che all'esterno. Da ultimo, inoltre, la famiglia "essendo parte di una comunità socio-culturale, condivide con questa sistemi di credenze che 
definiscono cosa è accettabile e desiderabile in termini di comportamenti, ruoli e rapporti familiari. Tali credenze concorrono alla determinazione delle dinamiche familiari che, a loro volta, contribuiscono a sviluppare e mantenere stereotipi, ideologie, valori condivisi sul piano sociale" (Fruggeri 1997, p.72).

Cecheremo dunque di mettere in luce, rispetto alla famiglia di questo terzo millennio, due aspetti particolari cha attengono da una parte alla dimensione strutturale e dall'altra a quella relazionale.

Qualunque forma presenti la famiglia, sappiamo da numerosi studi che sono stati compiuti, a partire da quello di Hill del 1949 sulle reazioni familiari alla separazione e riunificazione di un componente della famiglia a causa della guerra, che tutte le storie familiari sono attraversate da eventi critici:

- più o meno prevedibili (matrimonio, nascita, adolescenza, uscita di casa dai figli, pensionamento dei genitori, morte, separazioni familiari, disastri naturali, incidenti, malattie, gravi invalidità...)

- più o meno sfumati (se il momento della nascita è puntuale e circoscritto, il passaggio dell'infanzia all'adolescenza è sempre meno individuabile, per la mancanza nella nostra società di riti di passaggio).

Il concetto di ciclo vitale della famiglia è stato elaborato all'interno di una prospettiva interçisciplinare, l'approccio evolutivo allo studio della famiglia, già a partire dagli anni cinquanta.

Solo agli inizi degli anni Settanta, tuttavia, si può ritrovare il suo impiego nella pratica clinica.

Mc Goldrick e Carter (1987) utilizzano l'approccio evolutivo e il concetto di ciclo vitale per la comprensione sia della famiglia "normale" che di quella patologica. Viene adottata una prospettiva multigenerazionale per la spiegazione delle dinamiche familiari e la famiglia nucleare è vista come sottosistema che interagisce all'interno di un più vasto sistema trigenerazionale.

$\mathrm{L}$ 'affermare che sono in gioco almeno tre generazioni porta a postulare (Scabini, 1985, p. 85) «[...] l'esistenza di un asse verticale lungo il quale vengono trasmessi di generazione in generazione i modelli di relazione e di funzionamento [...]», e uno orizzontale che raccoglie gli «[...] stress che la famiglia incontra nel proprio cammino attraverso il tempo, adattandosi, più o meno attivamente, ai cambiamenti e alle modificazioni provocate dal superamento dei vari stadi». 
Su quest'ultimo asse vengono ricondotte anche tutte quelle tensioni derivate da accadimenti assolutamente imprevedibili e imputabili sia a eventi accidentali (morti, malattie, handicap) sia derivanti da fattori legati al sistema sociale (crisi economiche, trasferimenti, emigrazioni ecc.).

Le considerazioni proposte consentono di tenere presenti da un lato gli aspetti intergenerazionali dello sviluppo familiare e dall'altro gli influssi degli eventi legati al contesto socio-ambientale, sia nello studio dei processi che caratterizzano l'evoluzione della famiglia "normale", sia nella i momenti più significativi comprensione delle situazioni problematiche.

Noi sappiamo che questi eventi non determinano automaticamente una riorganizzazione, ma provocano turbolenza, disorganizzazione iniziale, crisi appunto come separazione da vecchie caratteristiche organizzative, perdita di una modalità di legame precedente, di un ruolo, di una rappresentazione di sè e dell'altro.

L'evento critico è un punto osservabile di un processo continuo di cambiamento di sviluppo, da cui possono derivare strategie evolutive diversificate, funzionali e disfunzionali.

Alcune analisi estremamente interessanti, compiute a livello sociologico in Italia, sottolineano come la differenza in ampiezza e varietà delle strutture familiari in Italia permase fino a tutta la prima metà del XX secolo, come conseguenza anche del lento e tardivo processo di industrializzazione e come i primi segnali di discontinuità nei comportamenti familiari possano farsi risalire proprio a questi anni (Saraceno,1998).

La rivoluzione sessuale, un non più esclusivo orientamento verso i figli, una nuova importanza attribuita al benessere ed alla felicità della coppia, la crescita dell'instabilità coniugale e infine il cambiamento del ruolo e della posizione sociale delle donne nella società sono fenomeni che, intrecciandosi alle profonde trasformazioni a livello di stratificazione sociale, di sviluppo economico e di modelli di consumo, si raffrozarono a vicenda, producendo cambiamenti profondi nel modo di fare famiglia.

L'assunto di base su cui si è costituita buona parte della sociologia della famiglia che nel passaggio da società semplici e da formazioni storico sociali tradizionali a società complesse e formazioni storicosociali moderne, la famiglia da estesa diventa nucleare modificandosi sia nella struttura che nelle funzioni, se considerato come prodotto assoluto di una istanza evoluzionistica, evidenzia tutta la sua debolezza. 
Ogni formazione storico-sociale è caratterizzata dalla compresenza di più forme familiari, alcune delle quali possono essere quantitativamente più incidenti $\mathrm{e}$ in questo senso è indubbio che attualmente la famiglia modale è la famiglia nucleare, ma è anche vero che molteplici risultano essere le forme di organizzazioni del quotidiano che si esternano nella famiglia (Donati- Di Nicola, 1989).

Dal punto di vista della struttura, possiamo individuare la seguente "modulazione" nelle forme familiari :

\section{Famiglia unipersonale}

Si tratta di una forma impropria, in quanto formata da un solo soggetto che occupa una unità abitativa. E' una forma familiare, tipica della società industriale e destinata ad avere un'incidenza percentuale sempre maggiore, sia per l'allungamento della vita (anziani, o meglio prevalentemente anziane che vivono sole, spesso vedovi/e), sia per una maggior indipendenza economica (legata alla fruizione di trattamenti pensionistici) che per il prevalere di modelli culturali che delegittimano forme di coabitazione tra nuclei e soggetti appartenenti a generazioni diverse. Inoltre, si possono trovare in questa situazione, soggetti relativamente giovani che si allontanano dal proprio nucleo familiare per mobilità territoriale legata al lavoro o per scelte culturali di indipendenza ed autonomia da parte dei cosiddetti single, o per risoluzione di precedenti legami matrimoniali.

\section{Famiglia di coppia}

In questa struttura possiamo collocare le coppie anziane e quelle giovani senza figli, le coppie non coniugate per scelta o per necessità e quelle che sperimentano un periodo di vita in comune prima del matrimonio. Anche questa forma risente delle trasformazioni socioculturali sopra accennate.

\section{Famiglia nucleare}

Dentro questo grande contenitore di famiglie formate da un solo nucleo con relativi figli, si possono trovare delle tipologie particolari di notevole interesse. Oltre alla più idealtipica coppia di genitori coniugati con figli non emancipati, si possono trovare le cosiddette famiglie nucleari lunghe formate dai genitori con figli adulti già indipendenti dal punto di vista economico che rimangono in casa o vi tornano dopo un'esperienza matrimoniale o di coppia fallita. E' una struttura relazionale al cui interno si trovano difficoltà rispetto alla risoluzione del legame genitori-figli. Figli adulti continuano a far riferimento ad una struttura di servizi gestita spesso da genitori anziani, dove non è 
chiaro chi riveste ruoli decisionali, chi sia dipendente affettivamente, chi non ha autonomia psicologica, chi vive in una situazione di comodo. La famiglia basata sulla convivenza, soprattutto quando questa derivi da una scelta esprime un'opzione culturale rispetto al prevalere della dimensione affettivo-relazionale versus la legalizzazione del rapporto che è ritenuta secondaria se non addirittura antagonista. E' una modalità in grande espansione, complessa da analizzare e che se generalizzata richiederebbe una serie di interventi regolativi anche a livello del sistema sociale rispetto al mantenimento di garanzie minime di diritto nei confronti dei partners e dei figli. Le famiglie monogenitoriali, dette anche famiglie incomplete, ad esclusione di quelle derivanti dalla morte di uno dei due genitori o dalla presenza di un genitore nubile o celibe con figli, rappresentano il portato del superamento dell'indissolubilità del vincolo matrimoniale ed introducono nuova criticità nella gestione delle dinamiche relazionali, laddove su di un unico coniuge ricade la responsabilità di educazione, socializzazione e custodia di figli piccoli con la presenza, sullo sfondo, di un ex partner, in molti casi con un ruolo di controllo.Complementari rispetto a queste situazioni sono le forme di famiglia mucleare ricostituita, in cui confluiscono sia i figli nati dai precedenti matrimoni di uno o di entrambi i partner, sia gli eventuali figli che possono nascere dalla nuova unione. La configurazione relazionale della famiglia ricostituita comporta un problema di non facile soluzione quanto alla nomenclatura per denominare i nuovi padri, le nuove madri e i fratelli/sorelle acquisiti e questo è espresivo di uno stato di disagio che gli studiosi avvertono nei figli nelle coppie ricostituite.In queste situazioni, si rivela sovente una difficoltà a stabilire confini e legami, indice di una identità più incerta e confusa rispetto a quella che si manifesta nelle famiglie nucleari di prime nozze. La situazione si aggrava proporzionalmente al numero dei possibili matrimoni successivi, portando la rete di parentela ad una tale complessità da generare seri problemi di identità in tutti i membri della parentela (di sangue e legale) che si sono intrecciati nel corso degli eventi di congiunzione e disgiunzione, con particolare riguardo ai bambini che si trovano in fase di costruzione di un'identità autonoma (Donati, 1998, p.273).

\section{Famiglia complessa}

Questa forma ha subito sicuramente una contrazione quantitativa, ma è ancora presente nelle tipologie attualmente rilevabili, non solo come sopravvivenza culturale di un passato ormai remoto, ma come strategia generale di organizzazione e riorganizzazione del quotidiano. Essa può esprimersi sia in forma multipla (compresenza di più nuclei completi di generazioni diverse) che in forma estesa (dove ad un nucleo 
si affiancano possibili ascendenti e collaterali) e dimostra, in numerosi contesti e in diverse fasi del ciclo di vita individuale, di potersi offrire come camera di compensazione per far fronte a nuove situazioni. Si pensi al divorziato che torna a casa con i figli o alla giovane coppia in cerca di abitazione che rimane a vivere con uno dei due nuclei d'origine $o$ ancora alla famiglia nucleare che accoglie al proprio interno un genitore anziano per accudirlo in quanto non più in grado di vivere da solo.

Complessivamente si può rilevare che sono cambiati i modi in cui le famiglie si formano, si trasformano si dividono e che vi sono alcuni fenomeni presenti nel contesto attuale:

- forte flessione del tasso di natalità

- prolungamento della permanenza dei giovani in famiglia

- elevamento dell'età al matrimonio

- aumento delle quote di celibi e nubili

- aumento del numero di giovani adulti che vivono soli

- aumento delle convivenze

- aumento dell'instabilità coniugale con conseguenti separazioni e divorzi che determinano la formazione di famiglie monoparentali o ricostruite, di famiglie unipersonali, ma anche di famiglie dai confini permeabili e con pendolarismo dei figli.

Accanto alle tipologie familiari sopra esposte non dobbiamo dimenticare di tenere in considerazione una variabile in più che, per lo meno in un contesto di immigrazione abbastanza recente può essere sottovalutata : la presenza di etnie diverse.

Se possono realizzarsi in situazione di regolarità famiglie unipersonali di giovani adulti, possiamo assistere a forme familiari atipiche di convivenza tra persone della stessa etnia o a famiglie di coppie e nucleari in cui il matrimonio o la convivenza si basano sull'incontro tra soggetti appartenenze a culture diverse, incontratisi nel territorio in cui hanno scelto di migrare ( coppie miste) o a ricongiungimenti familiari di famiglie spezzate in una certa fase del processo migratorio. E come considerare tutte le situazioni di irregolari presenti nel nostro territorio, dove la dimensione di relazione richiama le dinamiche tribali, in cui il minore può essere tenuto in "custodia" da fratelli, zii o cugini dove questi termini non hanno lo stesso significato di discendenza di sangue come nella nostra cultura? 
Nell'analisi della dimensione relazionale, risulta evidente come se è chiara la diffusione della famiglia nucleare in tutte le classi sociali, si può riscontrare la presenza di diverse strutture relazionali che hanno a che fare con i livelli di scolarizzazione, i rapporti col mercato del lavoro, le immagine simboliche di famiglia che sono state interiorizzate e la pluralizzazione degli stili di vita.

E' indubbio che nel passaggio dalla società tradizionale a quella contemporanea le relazioni familiari hanno lentamente acquisito i caratteri dell'affettività ed espressività come elemento caratterizzante sia il rapporto genitori-figli che la vita di coppia, ma questo non è un elemento sufficiente per omogeneizzare gli aspetti relazionali.

Può essere interessante delineare alcune delle tipologie prevalenti evidenziate attraverso questa analisi:

- Famiglia tradizionale parsonsiana, dove si assiste ad una netta divisione dei compiti tra i ruoli coniugali che risultano molto differenziati anche rispetto alla variabile del potere. Al marito-padre spetta il ruolo strumentale di acquisizione delle risorse per la famiglia e di rapporto con le agenzie extrafamiliari,nonchè la funzione di cinghia di trasmissione dei valori della società di riferimento, mentre alla moglie-madre tocca il ruolo espressivo che si manifesta nella stabilizzazione della personalità adulta e nella cura e allevamento dei figli.

- Famigliasimmetrica, in cui i ruoli coniugali risultano interscambiabili, sia per quanto riguarda $\mathrm{i}$ compiti interni al nucleo familiare che $\mathrm{i}$ rapporti con l'esterno.

- Famiglia a doppia carriera che si presenta egualitaria nelle relazioni coniugali e democratica nei confronti dei figli, con ambedue i coniugi impegnati in attività professionali extra-familiari.

- Famiglia di fatto, non fondata sul matrimonio con forte enfasi sull'autorealizzazione individuale, tendenzialmente ugualitaria nei rapporti coniugali, in cui la vita di coppia è centrale e si realizza come tale anche al di furoi della filiazione.

Accanto a queste tipologie che si possono considerare ormai classiche, sono compresenti altre modalità relazionali strettamente correlate all'appartenenza a determinate classi sociali.

Si possono, infatti ritrovare:

- nella classe operaia, un forte attaccamento all'esclusività sessuale nella coppia ed una rigida divisione dei ruoli tra i coniugi con modalità autoritaristiche nel rapporto con i figli 
- nelle classi sociali marginali una confusione nei ruoli genitoriali, la prevalenza di unioni di fatto, un forte autoritarismo nel maschio adulto, promiscuità tra mondo degli adulti e mondo dei bambini, precocemente iniziati alle esperienze di vita dei grandi

- nella classe media una maggior divisione delle responsabilità e dei compiti educativi e domestici tra i coniugi, rapporti tra genitori e figli improntati a modelli di autorità promozionale, talvolta ricadenti nel permissivismo ed un'elevata comunicazione affettiva nella coppia

- nelle classi sociali superiori un'enfasi sull'importanza della famiglia come simbolo dell'ascesa individuale e strumento per consolidare e potenziare le risorse del gruppo, una rigida divisione dei compiti tra i coniugi e un elevato controllo sul processo di socializzazione e di formazione delle nuove generazioni.

\section{ALCUNI NODI PROBLEMATICI}

Nella società contemporanea emergono forme familiari che sono il prodotto di un continuo spostamento dei confini fra ciò che viene considerato "pubblico" o "privato", sia nelle relazioni di coppia che in quelle genitori e figli.

Certamente si registra una radicalizzazione del processo di "nuclearizzazione" della famiglia con l'abbandono di modelli patriarcali e un isolamento piuttosto significativo dalla rete parentale, a cui si aggiunge una tendenza alla " privatizzazione" alla chiusura verso il mondo esterno per perseguire uno stile di vita soggettivizzato.

Il rapporto di coppia diventa più importante di quello con la parentela o la comunità e la ricerca del piacere personale e la soddisfazione dei due partner diventa il motivo centrale dell'unione.

Acquistano significato le spinte di carattere "espressivo" legate alla realizzazione del proprio sé anche all'interno dei confini familiari. Diminuiscono i matrimoni e si diffondono le convivenze o le libereunioni.

D'altra parte la famiglia diventa sempre più soggetto di interessi collettivi e oggetto di crescenti preoccupazioni pubbliche. Vengono ad assumere rilevanza pubblica, infatti comportamenti un tempo ritenuti come privati (violenza sessuale tra coniugi o l'abuso sui figli) e vengono sempre più chiamate in causa risorse familiari per attività di cura nei confronti di soggetti deboli (anziani, minori, handicappati) o 
ancora la famiglia diventa un"titolo" per godere di servizi, di diritti, di facilitazioni.

Questa compresenza di pressioni di segno opposto in una società che diventa sempre più complessa, rende difficile realizzare processi di mediazione tra esigenze diverse. La crisi della famiglia odierna si esprime nell'emergere di tensioni nelle relazioni coniugali (ad esempio con la rinegoziazione dei ruoli sessuali) e intergenerazionali (con la ridefinizione dei rapporti genitoriali in termini più democratici) che portano a fenomeni di instabilità e di ristrutturazione delle reti familiari.

Riteniamo utile analizzare questi due nodi della crisi familiare che riguardano in primo luogo le relazioni di genere (maschile e femminile) $\mathrm{e}$ in secondo luogo le relazioni intergenerazionali.

Rispetto alle relazioni di genere possiamo osservare come le conquiste del femminismo che per certi versi hanno liberato la donna da una logica di sottomissione e di sofferenza hanno prodotto una crisi in un equilibrio di vecchia data, introducendo una serie di effetti che vanno dalla difficoltà a proporre la trasmissione transgenerazionale dell'identità femminile in modo nuovo e coerente, alla incertezza nella relazione tra $i$ sessi, alla negazione di fatto delle pari opportunità ancorchè dichiarate a gran voce.

L'organizzazione familiare si e' modificata solo in gruppi ristretti della popolazione e soprattutto, come abbiamo visto nelle coppie di classe medio-alta con un certo grado di istruzione.

Se le aspirazioni di reciprocità fra i generi sono oggi più elevate di un tempo, i comportamenti effettivi richiamano spesso modelli tradizionali. Le donne che lavorano fuori casa devono affrontare in famiglia un secondo lavoro e, se hanno parenti da accudire, questa diventa spesso una terza occupazione.

La struttura tradizionale nelle relazioni tra i generi è delegittimata, ma quella nuova è poco tematizzata e i giovani si trovano impreparati ad affrontare la loro vita in coppia essendo inseriti in una cultura che non li ha abituati a fare progetti in questa direzione.

Si realizzano cosi' accomodamenti contingenti rispetto ai compiti dei coniugi che, laddove siano frutto di un generico egualitarismo, creano più difficoltà, ansia e fallimenti di quanto non portino ad una reale soddisfazione e maturazione di una nuova struttura dei ruoli capace di andare incontro alle esigenze del partner tenendo conto di quelle dell'altro. 
Certamente il contesto sociale (dagli orari dei negozi e dei servizi, all'organizzazione della scuola dei figli) non prevedono e non aiutano una partnership di genere.

Nuovi strumenti legislativi (vedi la possibilità estesa al maschio nella legge italiana di chiedere permessi per la nascita di un figlio) o di welfare possono aiutare e sostenere questo processo, ma si iniziano a vedere anche gruppi di famiglie che danno origine a nuove reti di vicinato, organizzazioni come le banche del tempo e le associazioni familiari che creano reti di solidarietà, consociandosi in forme cooperative, che valorizzano le differenze di genere senza imporre ruoli predefiniti a uomini e donne.

La crisi della famiglia si manifesta anche nei cambiamenti che si verificano a livello dei rapporti tra le diverse generazioni, come fattore strettamente collegato ai fenomeni demografici emergenti sopra analizzati.

- Si ha una piramide rovesciata con un solo discendente che deve farsi carico di piu' anziani;

- aumenta il tempo che i soggetti hanno da trascorrere in un determinato ruolo familiare, quando la genitorialità sia precoce e le distanze intergenerazionali si fanno molto corte;

- viceversa, quando la nascita dei figli viene ritardata si creano strutture intergenerazionali con vuoti di età. Genitori ormai adulti avranno tendenzialmente meno possibilità di generare figli, si troveranno con una maggiore distanza di età rispetto a questi, avranno figli ancora giovani e dipendenti nella fase della loro vecchiaia;

- la mancanza volontaria di figli o la riduzione al figlio unico porta,alla lunga, a strutture familiari prive di relazioni intergenerazionali. Nell'età anziana questo comporterà problemi rispetto alla ricerca di aiuti e sostegni;

- si diffondono relazioni intergenerazionali "ricostruite" a seguito di separazioni o divorzi che vanno a rompere non solo la continuità di coppia, ma a rendere difficili gli intrecci intergenerazionali (nonninipoti, generi e nuore con i suoceri...) e in compenso possono generare nuovi complessi reticoli. La caoticità di queste reti diventa maggiore nel caso si diventi genitori mediante tecniche di riproduzione artificiale con donatore esterno o mediante la gestazione dell'embrione affidata ad una terza persona.

Tutto questo comporta problemi. La compressione della generazione di mezzo fa' si che, ad esempio, una donna di 45 anni debba occuparsi 
di genitori e nonni anziani e, contemporaneamente, di figli non ancora autosufficienti o addirittura anche di nipoti.

I ruoli generazionali risultano allungati, producendo confusione e conflittualità. Figli adulti e maggiorenni restano a vivere in famiglia, con difficoltà a definire i confini dei ruoli legati alle età.

Quando si diventa adulti? Quando anziani?

Molti genitori giocano a restare giovani, molti figli non possono o non vogliono diventare grandi.

Non dimentichiamo che nella società odierna le barriere generazionali tra genitori e figli, quando non addirittura tra nonni e nipoti, tendono ad assottigliarsi, sia per la migliore qualità della vita che rende meno visibili le ingiurie del tempo, sia per la cura rispetto al proprio aspetto esteriore che per quell'atteggiamento di giovanilismo che tende a porre su un piano cameratesco di parità soggetti di età diverse che dovrebbero esercitare ruoli educativi e di sana autorità.

Padri e madri hanno bisogno di sapersi fare amici dei figli, entrando a poco a poco come compagni nel loro mondo, ma senza mimetizzarsi da ragazzi e ragazze. Non devono perdere la loro identità di adulti, altrimenti creano confusione nei figli.

Così come ai figli deve essere consentito di vivere la loro avventura, senza essere costretti a diventare adulti precocemente, ma neppure senza crogiolarsi in un ruolo di eterni Peter Pan che rifiutano di entrare nel mondo degli adulti, per anticipare per quanto possibile ciò che vorranno e dovranno essere.

Ancora influisce in questo espandersi della crisi, un'altra serie di elementi che attengono alla dimensione della sottocultura sociofamiliare e riguardano da una parte, lo spostamento dei figli da una posizione relativamente periferica nella famiglia ad una posizione di assoluta centralità, e, dall'altra il crescente prolungamento della fase di dipendenza dei figli dai genitori con il conseguente dilazionamento della responsabilizzazione di questi ultimi (si parla oggi della lunga adolescenza del giovane adulto). Tutto questo in una situazione in cui la centralità non e' frutto di un pensiero che vede il figlio come destinatario di un progetto, nell'ottica descritta dal poeta Kalil Gibran, in cui il genitore e' l'arco che scaglia la freccia verso il futuro, bensì il bambino è più spesso espressione, simbolo di un vissuto regressivo per i genitori.

Nella società attuale il figlio non è più considerato come forza lavoro, ma come indicatore del successo raggiunto, come status symbol 
da rivestire di oggetti firmati, da riempire di regali in sostituzione del tempo che non si sa, non si può o non si vuole dargli, come oggetto da esibire su cui proiettare le aspettative, come realizatore di tutti i bisogni incompiuti (non importa se non sono i suoi), come un giocattolo da rompere quando ci si stanca, come strumento di piacere (l'Italia è nelle medie internazionali per violenze intrafamiliari, e paradossalmente si parla di abuso sessuale come se fosse lecito un uso sessuale del minore) . Il bambino e' sempre di più desiderato, ma anche vissuto con paura $\mathrm{e}$ con sentimenti di ansia.

Mary Pipher (1995), psicologa e psicoterapeuta americana stigmatizza con forza la cultura degli Stati Uniti esprimendo una percezione disastrosa della vita della famiglia e della collettività negli Usa e affermando che "Per la prima volta nella storia i nostri figli non non vengono socializzati dai genitori... sono allevati, coltivati, educati dalla TV o da altri congegni “.

Cristopher Lasch (1975) e Robert Bly (1996), nel contesto americano, ma anche Mario Pollo (1997) in una interessante ricerca sui giovani oggi in Italia, riflettono sullo sradicamento dalla prospettiva temporale che caratterizza la nostra epoca. La TV, condiziona il linguaggio, la percezione del tempo. Cadendo la dimensione della narrazione, la possibilità di trasmettere cultura, valori, informazioni tra le diverse generazioni, viene meno, si perde il senso della continuità storica, il senso di appartenenza ad una successione di generazioni che affonda le sue radici nel passato e si proietta nel futuro.

La perdita del senso storico, comporta un lento dissolversi di qualsiasi interesse per la posterità e quindi una scarsa attenzione nei confronti dei bisogni delle nuove generazioni.

Nella famiglia si possono determinare effetti devastanti. Scrive Mara Selvini "Gli sforzi dei genitori moderni perché i loro figli si sentano amati e desiderati non riescono a nascondere un distacco di fondo quello di chi ha ben poco da trasmettere alle generazioni successiva e vede in ogni caso come prioritario il proprio diritto alla realizzazione di sé. Il distacco emozionale unito ai tentativi per convincere il bambino della sua posizione di privilegio all'interno della famiglia, costituiscono una base eccellente per avviarlo a formare una personalità narcisistica"(1998, p.209).

Al termine di queste riflessioni possiamo dire che le immagini e le previsioni che danno la famiglia come residuato strutturale a bassa rilevanza sociale sono assolutamente fuorvianti. 
$\mathrm{E}^{\prime}$ vero, questo si, come abbiamo potuto argomentare, che la crisi evidenzia tensione nelle relazioni coniugali e intergenerazionali, porta a fenomeni di instabilità e di ristrutturazione delle reti familiari e tutto questo fa emergere il constante processo di trasformazione che la porta a riorganizzarsi incessantemente.

La famiglia del resto, ancorché fatto primordiale, elemento fondante della società all'inizio della storia umana e' sostanzialmente relazione sociale che emerge come fenomeno prodotto dall'interazione tra le dimensioni intersoggettive (empatiche e comunicative) che la costituiscono come gruppo di mondo vitale e le relazioni strutturali (cioè quei vincoli che derivano dalle sub-culture e dalle aspettative del sistema sociale di appartenenza) che la costituiscono come istituzione sociale.

In tale relazione sono compresi e intrecciati sia gli aspetti relativi alla dimensione di senso (simbolico-intenzionale) che si sviluppa nell'intersoggettività, sia quelli di legame reciproco che si formano nelle aspettative della comunicazione a livello degli altri sottosistemi sociali (politico, economico, amministrativo, scolastico, religioso...) e relative istituzioni.

Per quanto possa essere gravoso, la famiglia deve rielaborare l'insieme di queste relazioni perché basa il suo funzionamento sulla comunicazione non solo interna, ma anche con l'esterno.

"Che una società complessa, quale quella che stiamo vivendo renda più difficile le connessioni, il rapporto tra coinvolgimento e distacco, i bilanciamenti fra aspetti associativi e dissociativi e modifichi in continuazione senso, simboli, aspettative, valori e norme della vita familiare, tutto ciò può aiutare a comprendere i problemi della famiglia odierna, la sua specificità, la sua continua crisi, ma anche la sua incessante riorganizzazione" (Donati, 1998, p.120).

\section{BIBLIOGRAFIA}

BLY, R. (1996): The sibling society. Addison Wesley Reading, Mass.

COOPER, D. (1972): La morte della famiglia. Einaudi, Torino.

DONATI, P.; DI NICOLA, P. (1989): Lineamenti di sociologia della famiglia. NIS, Roma.

DONATI, P. (1998): Manuale di sociologia della famiglia. Laterza, Bari.

FRUGGERI, L. (1997): Famiglie. Dinamiche interpersonali e processi psico-sociali. NIS, Roma.

FRUGGERI, L. (1998): Da contesto come oggetto alla contestualizzazione come principio di metodo, in "Connessioni", n. 3, pp.75-86.

FRUGGERI, L. (2001): I concetti di mononuclearità e plurinuclearità nella definizione di famiglia, in "Connessioni", 8, pp.11 ss. 
LASCH, C. (1981): La cultura del narcisismo. Bompiani, Milano.

MATURANA, H.; VARELA, F. (ed.or.1980): Autopoiesi e cognizioni. Marsilio, Venezia, 1985.

MCGOLDRICK, M., GERSON, F.G.: Genograms in "Family Assessment". Norton, New York, London, 1987.

PIPHER, M. (1995): Revivng Ophelia. Putnam Sons, New York.

POLLO, M. (1997): I giovani e la notte. Milella, Lecce.

SARACENO, C. (1996): Sociologia della famiglia. Il Mulino, Bologna.

SARACENO, C. (1998): Mutamenti della famiglia e politiche sociali in Italia. Il Mulino, Bologna.

SCABNI, E. (1985): L'organizzazione famiglia tra crisi e sviluppo. Angeli, Milano.

SELVINI PALAZZOLI, M. et al. (1998): Ragazze anoressiche e bulimiche. Cortina, Milano.

VON FOERSTER, H. (ed.or.1981): Sistemi che osservano. Astrolabio, Roma, 1987. 